\title{
Cathode-Sulfide Solid Electrolyte Interfacial Instability: Challenges and Solutions
}

\author{
Teerth Brahmbhatt ${ }^{1,2}$, Guang Yang ${ }^{1 *}$, Ethan C. Self ${ }^{1}$ and Jagjit Nanda ${ }^{1,2 *}$ \\ ${ }^{1}$ Chemical Sciences Division, Oak Ridge National Laboratory, Oak Ridge, TN, United States, ${ }^{2}$ Bredesen Center for \\ Interdisciplinary Research and Education, University of Tennessee Knoxville, Knoxville, TN, United States
}

All-solid-state batteries are a candidate for next-generation energy-storage devices due to potential improvements in energy density and safety compared to current battery technologies. Due to their high ionic conductivity and potential scalability through slurry processing routes, sulfide solid-state electrolytes are promising to replace traditional liquid electrolytes and enable All-solid-state batteries, but stability of cathode-sulfide solid-state electrolytes interfaces requires further improvement. Herein we review common issues encountered at cathode-sulfide SE interfaces and strategies to

OPEN ACCESS

Edited by:

Zhengcheng Zhang, Argonne National Laboratory (DOE), United States

Reviewed by:

Yong Yang, Xiamen University, China Nikhilendra Singh,

Toyota, United States

*Correspondence: Guang Yang yangg@ornl.gov Jagjit Nanda nandaj@ornl.gov

Specialty section:

This article was submitted to Electrochemical Energy Conversion and Storage,

a section of the journal Frontiers in Energy Research

Received: 08 June 2020

Accepted: 28 August 2020

Published: 22 October 2020

Citation:

Brahmbhatt $T$, Yang G, Self EC and Nanda J (2020) Cathode-Sulfide Solid

Electrolyte Interfacial Instability:

Challenges and Solutions.

Front. Energy Res. 8:570754.

doi: 10.3389/fenrg.2020.570754 alleviate these issues.

Keywords: sulfide solid-electrolyte, solid-state batteries, electrode/electrolyte interface, cathode/electrolyte interface, cathode coating

\section{INTRODUCTION}

Improvements in energy density, performance, and safety of battery technology are key to meeting international climate goals within the transportation and energy generation sectors (Deng et al., 2020). Regarding electrochemical energy storage, research focus has shifted from liquid to solid-state electrolytes (SSEs) to achieve these goals (Wang et al., 2015). Due to their high ionic conductivity and good processability, sulfide electrolytes are a very promising SSE for all-solid-state batteries (ASSBs) (Dudney et al., 2015). The primary challenge sulfide electrolytes face is their narrow chemical and electrochemical stability window, and when paired with high voltage cathodes and Li metal anodes, sulfide SSEs typically suffer from increasing interfacial resistance during cycling, thereby reducing ASSB capacity and cycle life (Lau et al., 2018; Li et al., 2020a). Chemical stability is the electrolytes' ability to withstand chemical contact with electrode materials, whereas the electrochemical stability window defines the voltage range where the SSE is neither oxidized nor reduced during ASSB operation. It is possible for sulfide based ASSBs to achieve high energy density and long cycle life even if the interface isn't thermodynamically stable, if the instability results in the formation of a kinetically stable passive film.

Although issues such as air-stability, moisture stability, dendrite growth, and thermal runaway have yet to be completely resolved, enabling sulfide ASSBs rests on stabilizing the electrode/ electrolyte interfaces (Muramatsu et al., 2011; Han et al., 2018; Chen et al., 2020; Zhang Z. et al., 2020). Practical issues pertaining to processability and scalability such as the adaptability of SSEs to current roll-to-roll manufacturing and the drops in ionic conductivity commonly observed during slurry processing must also be considered in lab and pilot scale research efforts (Xu et al., 2019). However, fundamental understanding of the cathode-sulfide electrolyte interface has been a tremendous challenge and remains elusive. Cathodes often include redox active materials, a polymer binder, conductive carbon additives, and the SSE, and each component and interactions among these 


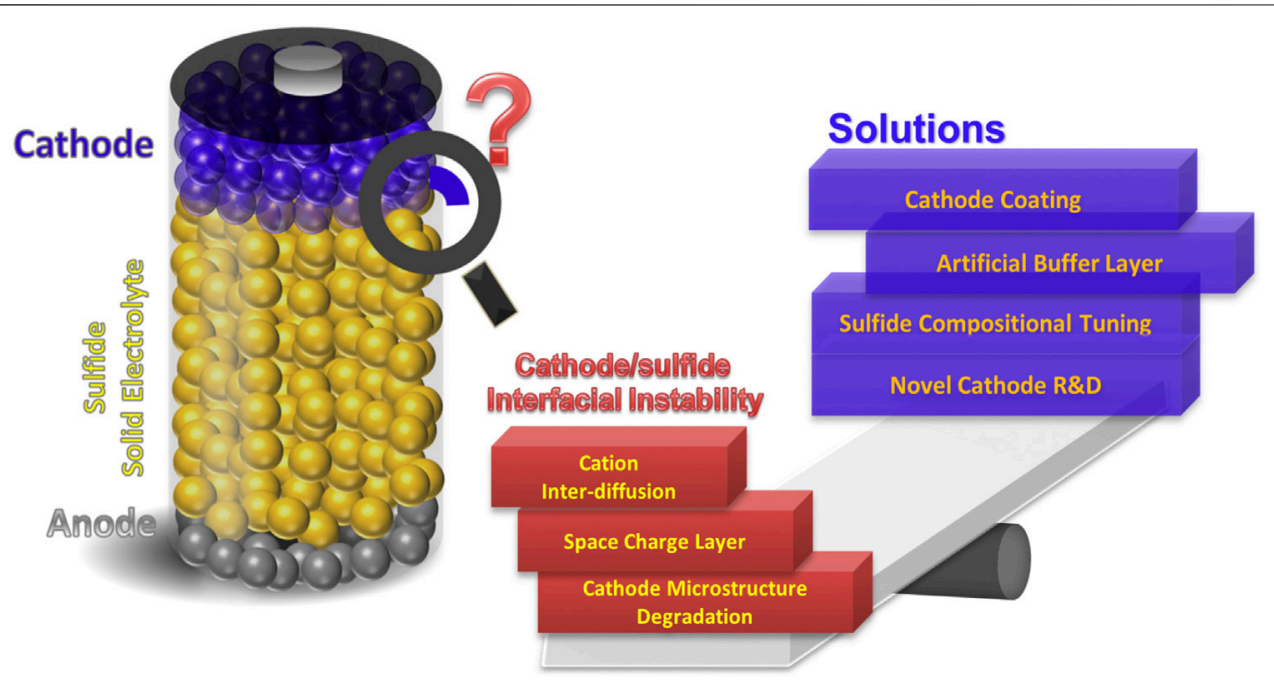

SCHEME 1 | Illustration of cathode/sulfide SSE interfacial instability issues and validated solutions.

components play critical roles in determining interfacial stability. As such, each component and their multifaceted interactions increases the technical complexity of characterizing the sulfide cathode interface (Pervez et al., 2019).

Cathode/sulfide SSE interfacial stability can involve broadly the chemical, electrochemical, and chemo-mechanical stability. For more comprehensive discussions, readers may resort to recently published reviews (Xiao et al., 2019b; Lau et al., 2018; Xia et al., 2019). This mini-review intends to provide a brief overview of the (electro)chemical instability at the sulfidecathode interface. Shown in Scheme 1, the interfacial instabilities include cation interdiffusion, space charge layer (SCL) formation, and microstructural degradation at the sulfide-cathode interface (Xia et al., 2019). Potential solutions to mitigate the interfacial instability will be discussed, including compositional tuning of sulfide electrolytes, active material coatings/buffer layers, and novel cathode materials with enhanced stability at the sulfide-cathode interface.

\section{INSTABILITY OF THE CATHODE-SULFIDE ELECTROLYTE INTERFACE}

One major challenge with sulfide electrolytes when using traditional Li-ion cathodes is cation interdiffusion during cycling. This is particularly true for high voltage transition metal oxides such as $\mathrm{LiCoO}_{2}$ (LCO), $\mathrm{LiNi}_{\mathrm{x}} \mathrm{Mn}_{\mathrm{y}} \mathrm{Co}_{(1-\mathrm{x}-\mathrm{y})} \mathrm{O}_{2}$ (NMC), and $\mathrm{LiNi}_{\mathrm{x}} \mathrm{Co}_{\mathrm{y}} \mathrm{Al}_{(1-\mathrm{x}-\mathrm{y})} \mathrm{O}_{2}$ (NCA) This issue was first observed at the LCO- $80 \mathrm{Li}_{2} \mathrm{~S} \cdot 20 \mathrm{P}_{2} \mathrm{~S}_{5}$ interface, where diffusion of cobalt from the cathode to the electrolyte was visible after initial charging. (Sakuda et al., 2010). The visualization of the Co was enabled by High-Angle Annular Dark-Field Scanning Transmission Electron Microscopy and Energy Dispersive $\mathrm{X}$-ray spectroscopy. Using computational methods, further studies showed that sulfide glasses and the (LGPS) electrolytes are prone to cation interdiffusion when placed against an LCO cathode (Woo et al., 2012; Richards et al., 2015). Co-P cation exchange at the $\mathrm{LCO} /$ sulfide interface has also been predicted from density functional theory (DFT) calculations (Haruyama et al., 2016). The major detrimental consequence of the Co interdiffusion is the formation of mixed conductors (e.g., CoS) which have lower ionic/higher electronic conductivities than the electrolyte itself. These species drive the electrolyte's continuous degradation at the interface, ultimately leading to large interfacial resistance and poor cycle life (Ito et al., 2014).

An additional driver of instability at the cathode/sulfide interface is the SCL formation. The SCL originates from the lithium chemical potential difference between the sulfide electrolyte and the cathode phases which results in $\mathrm{a} \mathrm{Li}^{+}$ depletion zone in the sulfide SSE near the interface (Haruyama et al., 2014). Although little information exists on the SCL formation at cathode/sulfide SE interfaces analogous studies have been performed with oxide-based ASSBs. De Klerk and Wagemaker examined $\mathrm{Li}_{1.2} \mathrm{Al}_{0.2} \mathrm{Ti}_{1.8}\left(\mathrm{PO}_{4}\right)_{3}$ (LATP) and (LLZO) electrolytes in contact with both LCO and graphite electrodes. The SCL was found to be $\leq 1 \mathrm{~nm}$ during cycling. The authors showed that the interfacial resistance attributed to the SCL varied based on lithium concentration differences between different compositions, and electrolytes with higher lithium ion concentrations were less likely to form a depletion layer which impedes $\mathrm{Li}^{+}$transport at the interface (de Klerk and Wagemaker, 2018). Results from some recent work contradict some of these findings, showing that SCLs can be larger than $1 \mathrm{~nm}$ in thickness. Cross-sectional transmission electron microscopy (TEM) images and electron holography data for the (LASGTP)-Cu electrode interface show the potential and lithium-ion gradients are at least $10 \mathrm{~nm}$ thick (Figures 1A,B) (Nomura et al., 2019). Although this work highlighted oxide based ASSBs, it illustrates how SCL formation and carrier depletion zones impact cathode-electrolyte interface stability. 
Given that sulfides are chemically less stable against LCO compared to LATP and LLZO, the SCLs could be thicker (Zhu et al., 2016; Chen et al., 2019). Much of the above discussion refers to works that attempt to build a general understanding of the SCLs effect on ASSB performance. However, it is clear that cathode and SE compositions play major roles in determining the impact of SCL formation on interfacial stability and cell performance. For this reason, detailed investigations with various sulfide SSEs and cathodes should be conducted to address this gap in the literature.

The third mechanism contributing to the cathode/sulfide interfacial instability is the interfacial microstructure (Takada, 2013). This view argued that the key to suppressing SCL formation and thereby charge carrier depletion zones is shielding the electrolyte from the cathode by lowering the electronic conductivity of the cathode's surface. Takada et al. utilized $\mathrm{Al}$ dopants to reduce the electronic conductivity of LCO near the surface. This resulted in capacity improvements at a rate of $5 \mathrm{C}$ from 10 to 80 when comparing undoped LCO with the doped equivalent in an LGPS based ASSB (Takada, 2013).

The cathode grain orientation also plays a significant role in cathode/sulfide electrochemo-mechanical stability. A recent study demonstrated that in cathode $/ \mathrm{Li}_{6} \mathrm{PS}_{5} \mathrm{Cl} / \mathrm{LiIn} \mathrm{ASSBs}$, the NCA cathodes with randomly-oriented grains were prone to severe particle disintegration during the first cycle, whereas NMC cathodes with radial grain orientations were capable of accommodating volume change and maintaining good solid/solid contact during cycling. These observations were enabled by operando electrochemical pressiometry and post-mortem electron microscopy. The authors concluded that compositional differences between NCA and NMC on the oxidative decomposition of $\mathrm{Li}_{6} \mathrm{PS}_{5} \mathrm{Cl}$ was marginal due to formation of similar SE decomposition products during charging (Jung et al., 2019).

The chemo mechanical compatibility and interconnectivity among cathode components plays an important role in determining the interfacial stability during cycling and overall cell performance. Using a model interface comprised of a $\left(\mathrm{Li}_{2} \mathrm{~S}\right)_{8}\left(\mathrm{P}_{2} \mathrm{~S}_{5}\right)_{2}\left(\mathrm{Ni}_{3} \mathrm{~S}_{2}\right)_{1}(\mathrm{LPN} 821) \mathrm{SSE}$ and $\mathrm{LiNbO}_{3}$ (LNO) coated NMC cathode, Choi et al. reported the pore size, tortuosity, volume, and connectivity among different components in the composite cathode. The quantification was enabled by a $3 \mathrm{D}$ reconstruction method combining focused-ion beam milling and SEM. The reconstruction revealed that the cathode and sulfide particle networks had significant overlap, but the polymer binder and conductive carbon agglomerated in micropores which limited their interconnectivity throughout the electrode (Choi et al., 2018). Shi et al. followed this work with microstructural modeling of the composite cathode and found that the limiting factor for high energy density was the ratio of cathode to SSE particle size, with the optimal value found to be (Shi et al., 2020). This work displays the importance of fabricating composite cathodes with high levels of interconnectivity to achieve target energy densities for sulfide ASSBs. Future points of exploration should focus on optimization of electronic and ionic conductivity within the bulk and at the surface of composite cathodes to improve the cycle life of sulfide based SSBs. Development of processing methods capable of producing dense cathodes with homogenous blends of active material, SE, and binder is also critical.

\section{ALLEVIATING CATHODE-SULFIDE INTERFACIAL INSTABILITY}

Several effective strategies to mitigate key issues associated with sulfide-cathode interfacial stability have been developed, including: 1) coatings and buffer layers on active materials, 2) sulfide compositional tuning, and 3) design of novel cathode materials. In this section, discussions regarding the coating methods include wet chemical, mechanochemical, and deposition-based techniques. A discussion of compositional tuning of electrolytes such as sulfur and phosphorous substitution will follow. Finally, a collection of emerging cathode materials such as layered transition metal sulfides, perovskites, and organic cathodes with improved compatibility with sulfide SSEs will be discussed.

Active material coatings and interlayers are among the most common strategies to stabilize the sulfide-cathode interface. An ideal coating promotes microstructural integrity during cycling by preventing formation of lithium depletion zones (i.e., SCLs) and detrimental side reactions, and prevent the occurrence of cation interdiffusion (Culver et al., 2019). For HVTMOs and sulfide electrolytes, the coating material should not be redox active between 2.8 and $4.2 \mathrm{~V}$ vs. $\mathrm{Li} / \mathrm{Li}^{+}$(Culver et al., 2018). Furthermore, these coatings should be good ionic conductors and electronic insulators to shield the electrolyte from oxidative decomposition against the active materials. The coating layer should also be elastic to accommodate volumetric change of the cathode during cycling.

As previously discussed, cation interdiffusion at the HVTMO cathode-sulfide interface leads to increased interfacial resistance and decreased cycle life. This issue can be effectively mitigated by using a coating such as $\mathrm{LiNbO}_{3}$ (LNO) on the cathode which prevents interdiffusion between $\mathrm{Co}$ and $\mathrm{P}$ (Haruyama et al., 2016). Current state-of-the-art ternary oxide coatings include and (Sakuda et al., 2010; Seino et al., 2011; Zhang W. et al., 2018). However, many of these oxide coatings are brittle and unable to accomodate cathode volume changes during cycling (Gruber et al., 2018). For example, a (LNTO) coating on LCO fractured after 300 cycles due to repeated cathode volume changes which ultimately led to Co diffusion into the SSE (Zhang W. et al., 2018).

While coatings have been successful in limiting cation interdiffusion, they also present the benefit of limiting harmful microstructural evolution during cycling at cathode-sulfide interface. Degradation of the cathode-sulfide interface microstructure, which can include cracking of individual cathode particles and near-surface phase changes, can increase interfacial instability and decrease cell performance. A recent study examined the microstructural evolution at the NMC-LGPS interface (Li et al., 2019b). The study compared the performance 


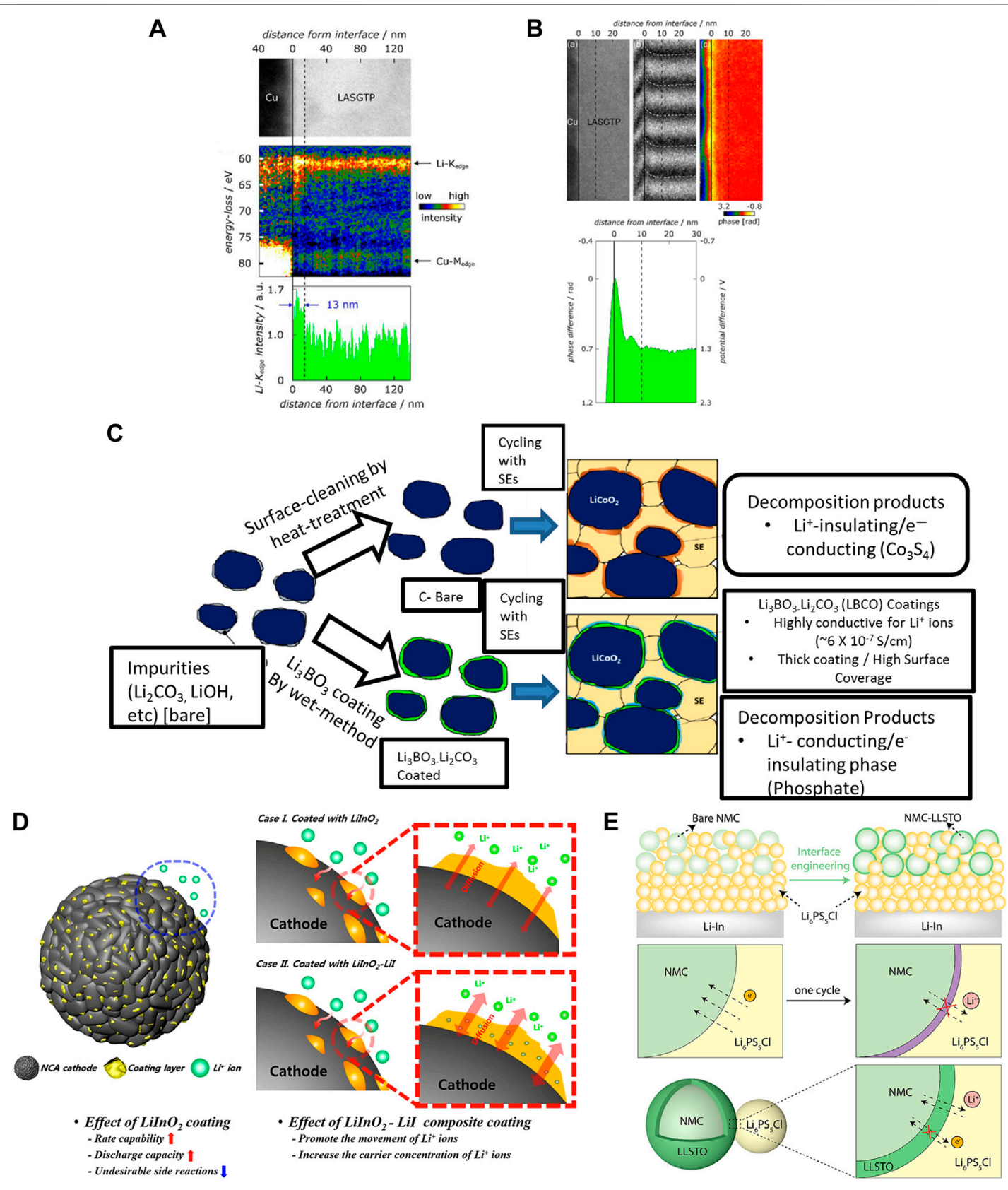

FIGURE 1 | (A) TEM and EELS images and resulting relative Li-K edge intensity profile of bulk SSE and SSE/Electrode interface (B) TEM and Electron Holograms of bulk and interface and resulting electrical potential profile. Reproduced with permission from John Wiley and Sons (Nomura et al., 2019). (C) Schematic of improvements in interface composition and durability due to $\mathrm{Li}_{3} \mathrm{BO}_{3}-\mathrm{Li}_{2} \mathrm{CO}_{3}$ coating reprinted with permission from (Jung et al., 2018). Copyright 2018 American chemical society. (D) Schematic displaying improvements in coating properties and performance as a function of Lil doping (Kwak and Park, 2019). (E) Schematic of (LLSTO) coating enabled all-solid-state battery and passivating interface reprinted with permission from (Cao et al., 2019) Copyright 2019 American chemical society.

of NMC cathodes with and without LNO coatings. During cycling, layered-to-rocksalt phase transformations near the surface were reported for uncoated NMC cathodes, whereas LNO-coated NMC experienced less structural disorder near the surface which promoted better capacity retention during cycling. To explore the underlying mechanisms, the authors used operando X-ray absorption near-edge spectroscopy to determine the interface degradation mechanism during cycling. It was shown that the surface NMC particles developed cracks during cycling which led to the diffusion of impurities into the secondary NMC particles and accelerated degradation of the NMC microstructure. Consequently, the cathode/sulfide interfacial resistance was increased, and the cell capacity faded over time (Li et al., 2019). The cell utilized 
for the operando X-ray absorption near-edge spectroscopy measurements is not representative of an optimized commercial cell, as the modified window into the cathode side of the cell could induce additional potential polarization. The operando study observed lower discharge plateaus when compared to the equivalent testing conditions in the standard cell. However, the goal of this study was to observe nonequilibrium phenomenon rather than optimize cell performance. Further studies should consider the impact that electrolyte composition on interphase and the microstructure degradation of the cathode. In addition, disordered rocksalt can be considered as a model cathode for studying microstructure degradation induced phase evolution on capacity fading during cell cycling.

If coatings are to play a key role in enabling sulfide ASSBs their scalability and compatibility with existing manufacturing and processing methods must be taken into consideration. Common solution-based methods to apply coatings include sol-gel, slurrybased, and spray-coating routes. Sol-gel processes which utilize alkoxide precursors followed by hydrolysis and polycondensation reactions have been used to produce a wide range of oxide-based cathode coatings (Kitaura et al., 2011; Liu et al., 2004). Slurry methods disperse cathode particles and coating precursors into a solvent following by drying and calcination steps as shown in Figure 1C. (Jung et al., 2018; Xu et al., 2019). Spray coating consists of preparing the coating material precursor solution and spraying it onto the active material particles, followed by heat treatments to achieve the desired form of the coating layer. Despite the scalability, these methods do not often allow for to precise control of the morphology, thickness, and homogeneity of the coating layer. For most lab scale applications, a combination of sol-gel and spray coating methods should suffice for screening of coating materials with various sulfide based solid-state chemistries.

In addition to solution-based routes, recent studies have utilized mechanochemical methods to coat active material particles. A study utilized resonant-acoustic dry coating of nanosized LNO and on NMC particles. When tested with a $\mathrm{Li}_{7} \mathrm{P}_{2} \mathrm{~S}_{8} \mathrm{I}$ SSE and Li-In counter electrode, the authors showed that the coated NMC had a capacity retention of $84 \%$ whereas the bare NMC had a capacity retention of $16.1 \%$ after 20 cycles at a current density of $\mathrm{mA} / \mathrm{g}$ (Kim et al., 2020). Impact blending is another effective mechanochemical method to apply cathode coatings. Nakamura et al. recently showed that this method could be used to form a core-shell layer of $\mathrm{Li}_{3} \mathrm{PS}_{4}$ (LPS) on LNO-coated NMC particles which resulted in higher ionic and lower electronic conductivities at the cathode-electrolyte interface compared to an untreated NMC electrode (Nakamura et al., 2020). Despite the promising results obtained with mechanochemical methods, scalability issues associated with these coating strategies must still be addressed.

Thin-film deposition methods such as atomic layer deposition (ALD) and pulsed laser deposition (PLD) represent another potential pathway to coat cathode particles. ALD has the advantage of ease of control in composition, thickness, and conformality of the coating layer on cathode particles. However, one of the major drawbacks of this method is the limited number of ALD precursors (Wen et al., 2016). PLD is another method used to produce tunable coatings on active materials (Yubuchi et al., 2016). Compared to ALD, PLD has decreased control over homogeneity. In addition, the film thickness generated by PLD is on the order of $50-1,000 \mathrm{~nm}$, whereas that of ALD is on the scale of $0.1-1 \mathrm{~nm}$. The increased thickness of the PLD coatings may impede $\mathrm{Li}^{+}$transport at the interface.

Composition of the cathode coating plays a critical role in the performance of sulfide-based SSBs. Some recent studies have unveiled new coating materials (e.g., lithium halides) as promising potential successors to LNO. A high-throughput computational screening study found fluorides and chlorides to be both chemically and electrochemically stable against both sulfides and NMC over wide potential ranges (Xiao et al., 2019a). A study recently applied an iodide-based coating, depicted in Figure 1D, to NCA cathodes paired with a sulfide SSE. The coating layer improved the cyclability significantly (Kwak and Park, 2019). A recent work employed $\mathrm{Li}_{3} \mathrm{InCl}_{6}$ (LIC) as a bulk electrolyte to form an NMC/LIC/ LGPS/In cell which exhibited stable performance over 70 cycles. This work shows that LIC is a promising material to investigate as a cathode coating for sulfide based ASSBs because an LGPS phase was used as a protective layer against the negative electrode for the LIC bulk (Li et al., 2019a). Wang et al. recently reported a coating of LIC on LCO particles improved the performance of an ASSB containing antiperovskite SSE (Wang C. et al., 2020). In addition to halide coatings, oxide, borate, and phosphate coatings may stabilize cathode/sulfide SSE interfaces. For example, conformal coatings on NMC particles drastically improved the rate performance and cycling stability as shown in Figure 1E (Cao et al., 2019). Borate and phosphate such as $\mathrm{LiBa}\left(\mathrm{B}_{3} \mathrm{O}_{5}\right)_{3}$, and $\mathrm{LiTi}_{2}\left(\mathrm{PO}_{4}\right)_{3}$ represent promising classes of cathode coatings due to their good chemical and electrochemical stability against both HVTMO cathodes and sulfide electrolytes (Xiao et al., 2019a). The coating materials, particularly those in the phosphate family include $\mathrm{AlPO}_{4}$ and $\mathrm{Li}_{1.3} \mathrm{Al}_{0.3} \mathrm{Ti}_{1.7}\left(\mathrm{PO}_{4}\right)_{3}$ have been explored in traditional lithium-ion batteries with liquid electrolytes (Cho et al., 2003; Li et al., 2019). Further work should focus on material compositional tuning to balance mechanical and ionic transport properties of the coating layer.

Compositional tuning of the sulfide SSE is another strategy to stabilize the sulfide-cathode interface. Two major doping strategies are used to improve the sulfide SSE chemical and electrochemical stability, including 1) oxygen substitution of sulfur and 2) partial or full substitution of phosphorus by atoms such as $\mathrm{Ge}, \mathrm{Si}, \mathrm{Sn}, \mathrm{As}$, and $\mathrm{Sb}$ using the corresponding soft acid. Recent work by Zhang et al. showed that doping the tetrahedra $\mathrm{PinLi}_{14} \mathrm{P}_{2} \mathrm{Ge}_{2} \mathrm{~S}_{16}$ with oxygen improves the ionic conductivity, moisture resistivity, and oxidative stability (Zhang B. et al., 2020). This work replaced the original tetrahedra in LGPS with yet maintained the composition of the original $\mathrm{GeS}_{4}$ to form $\mathrm{Li}_{14} \mathrm{P}_{2} \mathrm{Ge}_{2} \mathrm{~S}_{8} \mathrm{O}_{8} . \mathrm{Sb}$ and $\mathrm{Sn}$ substitution in LGPS and LPSCl SSEs has also been shown to improve ionic conductivity, cycle life, and moisture stability (Liang et al., 2020; Zhao et al., 2020). The examples provided here are only the beginnings of the potential advancements that can be made through compositional tuning to improve the ionic 
conductivity and stability of sulfide electrolytes with HVTMO cathodes. The ideal compositional tuning strategy may involve simultaneous use of the above paths in conjunction with efforts to make advantageous substitutions on Li sites (Rajagopal and Ryu, 2020). This is due to the balance that will be needed in using substitutions of $\mathrm{P}$ to improve the air and moisture stability, while using substitution of $\mathrm{S}$ with $\mathrm{O}$ to improve the electrochemical stability of the electrolyte with HVTMO cathodes. Further work should look at designing studies that utilize both computational and experimental methods to optimize the tradeoffs and benefits presented by each of these doping strategies when used in conjunction.

Development of new cathode materials and blending methods are an additional strategy to improve cathode-sulfide SE compatibility. Recent studies have shown that by blending two HVTMO types of cathode, the resulting mechanical, and (electro) chemical stability of the cathode with sulfide SSEs could be improved (Umeshbabu et al., 2019; Li et al., 2020b). For example, Koerver et al. showed that blending two types of cathodes, with one expanding (LCO) while the other (NMC) contracts upon delithiation, results in a composite cathode with much lower net volume change. This mitigated particle-particle contact loss at the cathode/sulfide interface and thus boosted cell performance (Koerver et al., 2018). While the Koerver et al. work is the first example of using blended cathodes within ASSBs the approach of blending active materials has been employed in traditional Li-ion battery research for decades (Chikkannanavar et al., 2014). These concepts should be expanded to balance mechanical properties across the entire stack as to accommodate net volume changes at both electrodes.

New lithium rich layered transition metal sulfides are promising cathode materials, which are inherently more chemically stable with sulfide electrolytes compare to their HVTMO counterparts. A recent study examined cathodes using a liquid electrolyte and lithium metal anode. Such cathodes exhibited reversible capacities up to and operating voltage ca. 2.5-3.0 V depending on the composition (FlamaryMespoulie et al., 2020). These materials have also recently been applied to sulfide based ASSBs Marchini and coauthors developed a $\mathrm{Li}_{1.13} \mathrm{Ti}_{0.57} \mathrm{Fe}_{0.3} \mathrm{~S}_{2}$ cathode which showed good cyclability in a cathode + LPS composite/LPS/Li solid-state cell. The cathode/ electrolyte interfacial resistance was between $25-35 \Omega \mathrm{cm}^{2}$ comparable to those of a HVTMO cathode even with a buffer layer coating. When cycled from 1.8 to $3.0 \mathrm{~V}$ vs. $\mathrm{Li} / \mathrm{Li}^{+}$the cell exhibited a reversible capacity of $214 \mathrm{mAhat} \mathrm{C} / 50$ over 10 cycles (Marchini et al., 2020). The slow cycling rate (C/50) was necessary to prevent short circuiting from unstable $\mathrm{Li}$ growth which occurred at higher current densities. Notably, practical ASSBs should enable stable Li plating/stripping at current densities on the order of $1 \mathrm{~mA} / \mathrm{cm}^{2}$.

In addition to lithium rich layered transition metal sulfides, other classes of cathode materials including perovskite and organic species have attracted significant attention for use in ASSBs. A 2D-hybrid halide perovskite + Vapor Grown Carbon | LPS | Li-In ASSB showed a reversible capacity of after 30 cycles and a low interfacial resistance between $10-26 \Omega \mathrm{cm}^{-2}$ before and after cycling. This cathode-sulfide interfacial resistance is lower than that of common coated HVTMO cathodes in ASSBs which after an initial cycle can have an interfacial resistance of 125.6-204.2 $\Omega \mathrm{cm}^{-2}$ (Fujii et al., 2019; Li et al., 2020a). The hybrid halide perovskite's high ionic conductivity also avoided the need for solid electrolyte powder in the cathode. This in turn increased the active material loading and improved cathode utilization during cycling. Redox-active organic species have unique mechanical properties such as superior reformability when compared to ceramic electrode materials and tunable redox potentials which make them attractive for use in ASSBs. For example, a 4-(phenylazo) benzoic acid lithium salt + LPS + carbon | LPS| Li ASSB showed stable performance with an initial discharge capacity of $120 \mathrm{mAh}$. Although a drastic drop of the Coulombic efficiency was observed due to the cell being discharged to $1.0 \mathrm{~V}$ vs. $\mathrm{Li} / \mathrm{Li}^{+}$, which is below the $1.71 \mathrm{~V}$ vs. $\mathrm{Li} / \mathrm{Li}^{+}$reduction potential of the sulfide electrolyte this in turn leads to decomposition of the electrolyte and the resulting low initial Coulombic efficiency upon further cycling the Coulombic efficiency approached 100\% during long-term cycling (Luo et al., 2018). The authors indicated that the high coulombic efficiency was enabled by ionic bonding between the cathode's carboxylic acid groups and $\mathrm{Li}^{+}$cation in the LPS, ultimately mitigating the loss of contact due to cathode volume changes during cycling. The challenges faced by this strategy stem primarily from the early onset of sulfide decomposition at $1 \mathrm{~V}$ vs. $\mathrm{Li} / \mathrm{Li}^{+}$when in contact with the cathode. However, these results still represent promising future development directions if the results are considered in the context of polymer interlayers often used with ceramic based ASSBs (Wang L. et al., 2020; Jena A. et al., 2018). If the ionic bonding capability of carboxylic acid groups and $\mathrm{Li}^{+}$cations can be harnessed and explored through functionalization of other types of SSEs such as poly (ethylene oxide) (PEO), the interfacial mechanical stability of sulfide electrolytes could be improved to enable long cycle life ASSBs.

A unique strategy for studying the interfacial effects between oxide and sulfide-based components is the construction of a hybrid cell. In a recent study conducted by Naguib et al. a hybrid cell composed of a sulfur carbon cathode, LLZO, and lithium metal anode was constructed. The porous cathode was infiltrated with an organic liquid electrolyte which decreased the otherwise high interfacial resistance observed for solid-solid interfaces. In this configuration, LLZO prevented polysulfide shuttling and enabled an initial discharge capacity of $1,154 \mathrm{mAh} / \mathrm{g}$ which faded to $604 \mathrm{mAh} / \mathrm{g}$ after 50 cycles at $100 \mathrm{~mA} / \mathrm{g}$ (Naguib et al., 2019). In another approach, mechanical constrictions in the form of core-shell geometries can improve interfacial stability by widening the electrochemical stability window of the SSE (Fitzhugh et al., 2019a). The core in these samples is a crystalline LSPS or LGPS while the shell is the amorphous form of the compound in which the sulfur composition has been decreased and the silicon or germanium composition increased. Fitzhugh et al. (2019b) have reported expanded electrochemical stability windows for LGPS and $\mathrm{Li}_{0.54} \mathrm{Si}_{1.74} \mathrm{P}_{1.44} \mathrm{~S}_{11.7} \mathrm{Cl}_{0.3}$ (LSPS) electrolytes by employing a combination of mechanochemical, high temperature, and sonochemical synthesis techniques to create these core-shell structures. Although these materials show great promise, 
potential scalability issues have not yet been addressed for the utilized synthesis methods. (Prasad and Dalvi, 2020). Another key property to consider for the improvement of sulfide based ASSB performance is increasing the critical current density. One such study utilized an aramid fiber to form a sulfide-fiber composite electrolyte which showed an improvement in critical current density from $200 \mu \mathrm{A} / \mathrm{cm}^{2}$ for the sulfide sample to $400 \mu \mathrm{A} / \mathrm{cm}^{2}$ for the sulfide-aramid composite (Yersak et al., 2019). The focus on manipulating mechanical properties of the electrolyte are a key area for future exploration, in addition the above methods the use of solid electrolyte fillers and infiltration solutions present a promising path toward improved energy density and cycle life.

As discussed above, the number of strategies available to potentially alleviate cathode-sulfide interfacial stability has grown tremendously over the past decade. To better understand the effects of cathode coatings, SSE compositional tuning, and new cathode materials on cathode-sulfide interfacial stability, it is desired to utilize in-operando methods to study buried interfaces in ASSBs (Wenzel et al., 2015). For example, operando X-ray Photoelectron Spectroscopy (XPS) was recently used to study the evolution of LPS/Li interface. The experiment revealed initial formation of $\mathrm{Li}_{2} \mathrm{~S}$ and $\mathrm{Li}-\mathrm{P}$ phases followed by the formation of Li-O phases due to oxygen contamination present at the sample surface and finally $\mathrm{Li}^{0}$ (Wood et al., 2018). In addition to XPS, in-situ Raman spectroscopy is capable of observing the changes in the chemistry at the LPS/Au interface in an Au/LPS/Li cell during lithium plating and stripping. The tetrahedra within the LPS underwent a partially reversible conversion from $\mathrm{PS}_{4}{ }^{3-}$ to $\mathrm{P}_{2} \mathrm{~S}_{6}{ }^{4-}$ with $\mathrm{Li}_{2} \mathrm{~S}$ formation occurring simultaneously during the plating of lithium at the LPS/Au interface (Sang et al., 2017). In a recent work, two-dimensional exchange Nuclear Magnetic Resonance spectroscopy (2D-EXSY) was used to quantitatively measure the change in charge transfer resistance over the $\mathrm{Li}_{2} \mathrm{~S}$-LPSCl interface both during processing steps (mixing, milling, heating etc.) and during cycling of a $\mathrm{Li}_{2} \mathrm{~S} / \mathrm{LPSCl} / \mathrm{In}$ ASSB (Yu et al., 2017). Initial charge-discharge cycles were found to dramatically decrease the rate of charge transfer across the $\mathrm{Li}_{2} \mathrm{~S}$-LPSCl cathode-sulfide interface due to volume expansion of the active material particles and the presence of products from side reactions with low ionic conductivities ( $\mathrm{Yu}$ et al., 2017). As is it has been discussed when dealing with the study of interfaces or model systems in ASSBs, cell configurations must be designed to observe specific phenomena. These designs often result in cells that do not meet commercial performance benchmarks as is the case with the examples and methods described above. These deviations from the ideal ASSB include limited ability to apply stack pressure and difficulty in avoiding air exposure during testing. In addition, characteristics of ASSBs such as the limited exposure of the buried interface and the heterogeneous nature of the interphase, that is, often below the dimensional resolution of commonly available instruments greatly limit the ability to craft effective experimental designs. Most characterization techniques discussed focus only on a certain scale on a limited area. For example, XPS probes the scale of $10 \mathrm{~nm}$ in sample plane, Raman has a submicron spatial resolution, SEM has nm resolution and TEM can go down to nmatomic scale. The interfacial instability is initiated at the atomic scale, and its impact on the cell performance is in macro-scale $(\mathrm{mm}$ to $\mathrm{cm})$. To properly understand the cathode/interfacial instability necessitates multi-scale, multi-modal characterization techniques, and scale-bridging means. Furthermore, studies in the literature focus only on a limited area in one or two cells. Future studies of cathode-sulfide interfaces should focus on conducting studies with a larger array of samples to determine and establish a standardized acceptable error for a given technique.

\section{SUMMARY AND PROSPECTS}

In summary, sulfide-based SSEs are key enablers for next generation ASSBs with high energy density and low cost. The (electro)chemical instability of the cathode/sulfide SSE interface is a major bottleneck which hinders development of sulfide SSEs. The challenges associated with these materials include interdiffusion of transition metal ions, heterogeneous $\mathrm{Li}+$ distribution near the interface in the SCL, and microstructural evolution of the cathode and/or sulfide SE during cycling. To address these issues, strategies to improve the cathode/sulfide interfacial stabilities have been explored, including design of protective coatings on cathode, compositional tuning of the cathode and sulfide SSEs, and development of new cathodes with enhanced compatibility with the sulfide electrolytes. Future focus should be given to: i) identifying interfacial decomposition reactions and ii) structural and morphological evolution using characterization methods with multiscale spatial resolution, sensitivity, and multimodality. A proper understanding of the failure mechanisms during cycling due to cathode/SE interfacial instability should provide guidance for the development of protective coating layers, novel dopants, and other treatments to further improve the performance of SSBs with sulfide-based SEs. With regards to the development of novel coatings new technology is necessary to accelerate the material discovery process. The integration of computational chemistry with reaction dynamics, and charge transport at the electrode/ electrolyte interface is desired. Desirable artificial intelligence and machine learning algorithms are necessary to address the challenges including scale bridging, molecular screening, correlating structure-activity, and property to stabilize the cathode/SSE interface. In addition to the compositional tuning of existing sulfide electrolytes and the development of new phases a standardized testing procedure must be adopted for measuring the ionic conductivity, as the literature has shown this can vary for the same material across multiple research groups (Ohno et al., 2020). Similar such interlaboratory studies should be conducted for model cathode-sulfide systems to build an understanding of how interfacial measurements can vary due to variations in interpretation and technique across research groups.

In order for sulfide ASSBs to move toward commercialization cost estimates at various scales and determination of necessary targets must be communicated to the wider research community. Recent attempts at estimating the production cost at the cell level for sulfide based ASSBs has illuminated that material costs for 
sulfide electrolytes compared to the traditional $\mathrm{LiPF}_{6}$ based organic liquid electrolytes will be significantly higher $\$ 50 / \mathrm{kg}$ vs. $\$ 12 / \mathrm{kg}$ (Schmuch et al., 2018). However, follow-up analysis has shown that even with the higher electrolyte material cost, sulfide based ASSBs can still be cost competitive with liquid electrolyte cells due to lower processing costs driven by the omission of the electrolyte filling process and simplified formation procedure (Schnell et al., 2020). Future work should look to address the assumptions made in such cost models by validation of scaling and processing parameters at the pilot and industrial scales. Most of these studies did not take into account additional potential processing steps and material requirements such as oxide-based coatings and artificial SEIs when considering ASSBs featuring a Li metal anode. Similar analysis including these components would be a valuable addition to the literature and help guide the scale up and commercialization of this technology.

\section{AUTHOR'S NOTE}

This manuscript has been authored by UT-Battelle, LLC under Contract No. DE-AC05-00OR22725 with the U.S. Department of Energy. The United States Government retains and the publisher, by accepting the article for publication, acknowledges that the United States Government retains a non-exclusive, paid-up, irrevocable, worldwide license to publish or reproduce the published form of this manuscript,

\section{REFERENCES}

Cao, D., Zhang, Y., Nolan, A. M., Sun, X., Liu, C., Sheng, J., et al. (2019). Stable thiophosphate-based all-solid-state lithium batteries through conformally interfacial nanocoating. Nano Lett. 20 (3), 1483-1490. doi:10.1021/acs. nanolett.9b02678

Chen, R., Li, Q., Yu, X., Chen, L., and Li, H. (2019). Approaching practically accessible solid-state batteries: stability issues related to solid electrolytes and interfaces. Chem. Rev. 120, 6820-6877. doi:10.1021/acs.chemrev.9b00268

Chen, R., Nolan, A. M., Lu, J., Wang, J., Yu, X., Mo, Y., et al. (2020). The thermal stability of lithium solid electrolytes with metallic lithium. Joule 4 (4), 812-821. doi:10.1016/j.joule.2020.03.012

Chikkannanavar, S. B., Bernardi, D. M., and Liu, L. (2014). A review of blended cathode materials for use in Li-ion batteries. J. Power Sources 248, 91-100. doi:10.1016/j.jpowsour.2013.09.052

Cho, J., Lee, J.-G., Kim, B., and Park, B. (2003). Effect of $\mathrm{P}_{2} \mathrm{O}_{5}$ and $\mathrm{AlPO}_{4}$ coating on $\mathrm{LiCoO}_{2}$ cathode. Chem. Mater. 34 (45), 3190-3193. doi:10.1002/chin.200345018

Choi, S., Jeon, M., Ahn, J., Jung, W. D., Choi, S. M., Kim, J.-S., et al. (2018). Quantitative analysis of microstructures and reaction interfaces on composite cathodes in all-solid-state batteries using a three-dimensional reconstruction technique. ACS Appl. Mater. Interfaces 10 (28), 23740-23747. doi:10.1021/ acsami.8b04204

Culver, S. P., Koerver, R., Krauskopf, T., and Zeier, W. G. (2018). Designing ionic conductors: the interplay between structural phenomena and interfaces in thiophosphate-based solid-state batteries. Chem. Mater. 30 (13), 4179-4192. doi:10.1021/acs.chemmater.8b01293

Culver, S. P., Koerver, R., Zeier, W. G., and Janek, J. (2019). On the functionality of coatings for cathode active materials in thiophosphatebased all-solid-state batteries. Adv. Energy Mater. 9 (24), 1900626. doi:10. 1002/aenm.201900626

de Klerk, N. J. J., and Wagemaker, M. (2018). Space-charge layers in all-solid-state batteries; important or negligible? ACS Appl. Energy Mater 1, 5609-5618. doi:10.1021/acsaem.8b01141 or allow others to do so, for United States Government purposes. The Department of Energy will provide public access to these results of federally sponsored research in accordance with the DOE Public Access Plan (http://energy.gov/downloads/doepublicaccess-plan).

\section{AUTHOR CONTRIBUTIONS}

TB initiated the first draft. JN and GY mentored TB in structuring and organizing the manuscript. All authors contributed to writing/revising the manuscript.

\section{FUNDING}

This research was conducted at Oak Ridge National Laboratory, managed by UT Battelle, LLC, for the U.S. Department of Energy (DOE) and is supported by Asst. Secretary, Energy Efficiency and Renewable Energy (EERE), Vehicle Technologies Office (VTO) through the Advanced Battery Materials Research (BMR) Program.

\section{ACKNOWLEDGMENTS}

We are in debt to Dr. Frank M. Delnick for his fruitful discussion and revision on this article.

Deng, J., Bae, C., Denlinger, A., and Miller, T. (2020). Electric vehicles batteries: requirements and challenges. Joule 4 (3), 511-515. doi:10.1016/j.joule.2020.01. 013

N. Dudney, J. Nanda, and W. West (Editors) (2015). Handbook of solid state batteries. 2nd Edn. Singapore: World Scientific Publishing Company.

Fitzhugh, W., Wu, F., Ye, L., Su, H., and Li, X. (2019a). Strain-stabilized ceramicsulfide electrolytes. Small 15, e1901470. doi:10.1002/smll.201901470

Fitzhugh, W., Ye, L., and Li, X. (2019b). The effects of mechanical constriction on the operation of sulfide based solid-state batteries. J. Mater. Chem. A 7 (41), 23604-23627. doi:10.1039/c9ta05248h

Flamary-Mespoulie, F., Boulineau, A., Martinez, H., Suchomel, M. R., Delmas, C., Pecquenard, B., et al. (2020). Lithium-rich layered titanium sulfides: cobalt- and Nickel-free high capacity cathode materials for lithium-ion batteries. Energy Storage Mater. 26, 213-222. doi:10.1016/j.ensm.2019.12.033

Fujii, Y., Ramirez, D., Rosero-Navarro, N. C., Jullian, D., Miura, A., Jaramillo, F., et al. (2019). Two-dimensional hybrid halide perovskite as electrode materials for all-solid-state lithium secondary batteries based on sulfide solid electrolytes. ACS Appl. Energy Mater. 2 (9), 6569-6576. doi:10.1021/ acsaem.9b01118

Gruber, M., Leitner, A., Kiener, D., Supancic, P., and Bermejo, R. (2018). Incipient plasticity and surface damage in $\mathrm{LiTaO}_{3}$ and $\mathrm{LiNbO}_{3}$ single crystals. Mater. Des. 153, 221-231. doi:10.1016/j.matdes.2018.04.082

Han, F., Yue, J., Zhu, X., and Wang, C. (2018). ressing Li dendrite formation in $\mathrm{Li}_{2} \mathrm{~S}-\mathrm{P}_{2} \mathrm{~S}_{5}$ solid electrolyte by LiI incorporation. Adv. Energy Mater. 8 (18), 1703644. doi:10.1002/aenm.201703644

Haruyama, J., Sodeyama, K., Han, L., Takada, K., and Tateyama, Y. (2014). Space-charge layer effect at interface between oxide cathode and sulfide electrolyte in all-solid-state lithium-ion battery. Chem. Mater. 26 (14), 4248-4255. doi:10.1021/cm5016959

Haruyama, J., Sodeyama, K., and Tateyama, Y. (2016). Cation mixing properties toward Co diffusion at the $\mathrm{LiCoO}_{2}$ cathode/sulfide electrolyte interface in a solid-state battery. ACS Appl. Mater. Interfaces 9 (1), 286-292. doi:10.1021/ acsami.6b08435

Ito, S., Fujiki, S., Yamada, T., Aihara, Y., Park, Y., Kim, T. Y., et al. (2014). A rocking chair type all-solid-state lithium ion battery adopting $\mathrm{Li}_{2} \mathrm{O}-\mathrm{ZrO}_{2}$ coated 
LiNi0.8Co0.15Al0.05O2 and a sulfide based electrolyte. J. Power Sources 248, 943-950. doi:10.1016/j.jpowsour.2013.10.005

Jena, A., Meesala, Y., Hu, S.-F., Chang, H., and Liu, R.-S. (2018). Ameliorating interfacial ionic transportation in all-solid-state Li-ion batteries with interlayer modifications. ACS Energy Lett. 3 (11), 2775-2795. doi:10.1021/acsenergylett.8b01564

Jung, S. H., Kim, U. H., Kim, J. H., Jun, S., Yoon, C. S., Jung, Y. S., et al. (2019). Nirich layered cathode materials with electrochemo-mechanically compliant microstructures for all-solid-state Li batteries. Adv. Energy Mater. 10 (6), 1903360. doi:10.1002/aenm.201903360

Jung, S. H., Oh, K., Nam, Y. J., Oh, D. Y., Brüner, P., Kang, K., et al. (2018). $\mathrm{Li}_{3} \mathrm{BO}_{3^{-}}$ $\mathrm{Li}_{2} \mathrm{CO}_{3}$ : rationally designed buffering phase for sulfide all-solid-state $\mathrm{Li}$-ion batteries. Chem. Mater. 30 (22), 8190-8200. doi:10.1021/acs.chemmater. $8 \mathrm{~b} 03321$

Kim, Y.-J., Rajagopal, R., Kang, S., and Ryu, K.-S. (2020). Novel dry deposition of $\mathrm{LiNbO}_{3}$ or $\mathrm{Li}_{2} \mathrm{ZrO}_{3}$ on $\mathrm{LiNi}_{0.6} \mathrm{Co}_{0.2} \mathrm{Mn}_{0.2} \mathrm{O}_{2}$ for high performance all-solid-state lithium batteries. Chem. Eng. J. 386, 123975. doi:10.1016/j.cej.2019.123975

Kitaura, H., Hayashi, A., Tadanaga, K., and Tatsumisago, M. (2011). Improvement of electrochemical performance of all-solid-state lithium secondary batteries by surface modification of $\mathrm{LiMn}_{2} \mathrm{O}_{4}$ positive electrode. Solid State Ion. 192 (1), 304-307. doi:10.1016/j.ssi.2010.08.019

Koerver, R., Zhang, W., de Biasi, L., Schweidler, S., Kondrakov, A. O., Kolling, S., et al. (2018). Chemo-mechanical expansion of lithium electrode materials - on the route to mechanically optimized all-solid-state batteries. Energy Environ. Sci. 11 (8), 2142-2158. doi:10.1039/c8ee00907d

Kwak, H. W., and Park, Y. J. (2019). Cathode coating using $\mathrm{LinnO}_{2}$-LiI composite for stable sulfide-based all-solid-state batteries. Sci. Rep. 9 (1), 8099. doi:10. 1038/s41598-019-44629-x

Lau, J., DeBlock, R. H., Butts, D. M., Ashby, D. S., Choi, C. S., and Dunn, B. S. (2018). Sulfide solid electrolytes for lithium battery applications. Adv. Energy Mater. 8 (27), 1800933. doi:10.1002/aenm.201800933

Luo, C., Ji, X., Chen, J., Gaskell, K. J., He, X., Liang, Y., et al. (2018). Solid-state electrolyte anchored with a carboxylated azo compound for all-solid-state lithium batteries. Angew. Chem. Int. Ed. Engl. 130 (28), 8703-8707. doi:10. 1002/ange.201804068

Li, L., Zhao, R., Xu, T., Wang, D., Pan, D., Zhang, K., et al. (2019). Stabilizing a high-voltage $\mathrm{LiNi}_{0.5} \mathrm{Mn}_{1.5} \mathrm{O}_{4}$ cathode towards all solid state batteries: a Li-AlTi-P-O solid electrolyte nano-shell with a host material. Nanoscale 11 (18), 8967-8977. doi:10.1039/c9nr01655d

Li, X., Jin, L., Song, D., Zhang, H., Shi, X., Wang, Z., et al. (2020a). $\mathrm{LiNbO}_{3}$-coated $\mathrm{LiNi}_{0.8} \mathrm{Co}_{0.1} \mathrm{Mn}_{0.1} \mathrm{O}_{2}$ cathode with high discharge capacity and rate performance for all-solid-state lithium battery. J. Energy Chem. 40, 39-45. doi:10.1016/j.jechem.2019.02.006

Li, X., Liang, J., Chen, N., Luo, J., Adair, K. R., Wang, C., et al. (2019a). Watermediated synthesis of a superionic halide solid electrolyte. Angew. Chem. Int. Ed. 58 (46), 16427-16432. doi:10.1002/anie.201909805

Li, X., Ren, Z., Norouzi Banis, M., Deng, S., Zhao, Y., Sun, Q., et al. (2019b). Unravelling the chemistry and microstructure evolution of a cathodic interface in sulfide-based all-solid-state Li-ion batteries. ACS Energy Lett. 4 (10), 2480-2488. doi:10.1021/acsenergylett.9b01676

Li, X., Sun, Q., Wang, Z., Song, D., Zhang, H., Shi, X., et al. (2020b). Outstanding electrochemical performances of the all-solid-state lithium battery using Nirich layered oxide cathode and sulfide electrolyte. J. Power Sources 456, 227997. doi:10.1016/j.jpowsour.2020.227997

Liang, J., Chen, N., Li, X., Li, X., Adair, K. R., Li, J., et al. (2020). $\mathrm{Li}_{10} \mathrm{Ge}\left(\mathrm{P}_{1-\mathrm{X}} \mathrm{Sb}_{\mathrm{x}}\right)_{2} \mathrm{~S}_{12}$ lithium-ion conductors with enhanced atmospheric stability. Chem. Mater. 32 (6), 2664-2672. doi:10.1021/acs.chemmater.9b04764

Liu, H., Wu, Y. P., Rahm, E., Holze, R., and Wu, H. Q. (2004). Cathode materials for lithium ion batteries prepared by solgel methods. ChemInform, 35 (37), 450-466. doi:10.1002/chin.200437233

Liu, L., Qi, X., Yin, S., Zhang, Q., Liu, X., Suo, L., et al. (2019). In situ formation of a stable interface in solid-state batteries. ACS Energy Lett. 4 (7), 1650-1657. doi:10.1021/acsenergylett.9b00857

Marchini, F., Saha, S., Alves Dalla Corte, D., and Tarascon, J. M. (2020). Li-rich layered sulfide as cathode active materials in all-solid-state Li-metal batteries. ACS Appl. Mater. Interfaces 12 (13), 15145-15154. doi:10.1021/acsami.9b22937

Muramatsu, H., Hayashi, A., Ohtomo, T., Hama, S., and Tatsumisago, M. (2011). Structural change of $\mathrm{Li}_{2} \mathrm{~S}-\mathrm{P}_{2} \mathrm{~S}_{5}$ sulfide solid electrolytes in the atmosphere. Solid State Ion. 182 (1), 116-119. doi:10.1016/j.ssi.2010.10.013
Naguib, M., Sharafi, A., Self, E. C., Meyer, H. M., Sakamoto, J., and Nanda, J. (2019). Interfacial reactions and performance of $\mathrm{Li}_{7} \mathrm{La}_{3} \mathrm{Zr}_{2} \mathrm{O}_{12}$-stabilized $\mathrm{Li}$-sulfur hybrid cell. ACS Appl. Mater. Interfaces. 11 (45), 42042-42048. doi:10.1021/ acsami.9b11439

Nakamura, H., Kawaguchi, T., Masuyama, T., Sakuda, A., Saito, T., Kuratani, K., et al. (2020). Dry coating of active material particles with sulfide solid electrolytes for an all-solid-state lithium battery. J. Power Sources 448, 227579. doi:10.1016/j.jpowsour.2019.227579

Nomura, Y., Yamamoto, K., Hirayama, T., Ouchi, S., Igaki, E., and Saitoh, K. (2019). Direct observation of a Li-ionic space-charge layer formed at an electrode/solid-electrolyte interface. Angew. Chem. 131 (16), 5346-5350. doi:10.1002/ange.201814669

Ohno, S., Bernges, T., Buchheim, J., Duchardt, M., Hatz, A.-K., Kraft, M. A., et al. (2020). How certain are the reported ionic conductivities of thiophosphatebased solid electrolytes? An interlaboratory study. ACS Energy Lett. 5 (3), 910-915. doi:10.1021/acsenergylett.9b02764

Pervez, S. A., Cambaz, M. A., Thangadurai, V., and Fichtner, M. (2019). Interface in solid-state lithium battery: challenges, progress, and outlook. ACS Appl. Mater. Interfaces 11 (25), 22029-22050. doi:10.1021/acsami.9b02675

Prasad, R., and Dalvi, S. V. (2020). Sonocrystallization: monitoring and controlling crystallization using ultrasound. Chem. Eng. Sci. 226, 115911. doi:10.1016/j.ces. 2020.115911

Rajagopal, R., and Ryu, K.-S. (2020). A novel Na substituted $\mathrm{Li}_{3-\mathrm{x}} \mathrm{Na}_{\mathrm{x}} \mathrm{PS}_{4}$ mixed ions solid electrolyte for all solid-state lithium batteries. Appl. Surf. Sci. 527, 146739. doi:10.1016/j.apsusc.2020.146739

Richards, W. D., Miara, L. J., Wang, Y., Kim, J. C., and Ceder, G. (2015). Interface stability in solid-state batteries. Chem. Mater. 28 (1), 266-273. doi:10.1021/acs. chemmater.5b04082

Sakuda, A., Hayashi, A., and Tatsumisago, M. (2010). Interfacial observation between $\mathrm{LiCoO}_{2}$ electrode and $\mathrm{Li}_{2} \mathrm{~S}-\mathrm{P}_{2} \mathrm{~S}_{5}$ solid electrolytes of all-solid-state lithium secondary batteries using transmission electron microscopy. Chem. Mater. 22 (3), 949-956. doi:10.1021/cm901819c

Sang, L., Haasch, R. T., Gewirth, A. A., and Nuzzo, R. G. (2017). Evolution at the solid electrolyte/gold electrode interface during lithium deposition and stripping. Chem. Mater. 29 (7), 3029-3037. doi:10.1021/acs.chemmater.7b00034

Schmuch, R., Wagner, R., Hörpel, G., Placke, T., and Winter, M. (2018). Performance and cost of materials for lithium-based rechargeable automotive batteries. Nat. Energy 3 (4), 267-278. doi:10.1038/s41560-018-0107-2

Schnell, J., Knörzer, H., Imbsweiler, A. J., and Reinhart, G. (2020). Solid versus liquid-A bottom-up calculation model to analyze the manufacturing cost of future high-energy batteries. Energy Technol. 8 (3), 1901237. doi:10.1002/ente. 201901237

Seino, Y., Ota, T., and Takada, K. (2011). High rate capabilities of all-solid-state lithium secondary batteries using $\mathrm{Li}_{4} \mathrm{Ti}_{5} \mathrm{O}_{12}$-coated $\mathrm{LiNi}_{0.8} \mathrm{Co}_{0.15} \mathrm{Al}_{0.05} \mathrm{O}_{2}$ and a sulfide-based solid electrolyte. J. Power Sources 196 (15), 6488-6492. doi:10. 1016/j.jpowsour.2011.03.090

Shi, T., Tu, Q., Tian, Y., Xiao, Y., Miara, L. J., Kononova, O., et al. (2020). All-solidstate batteries: high active material loading in all-solid-state battery electrode via particle size optimization. Adv. Energy Mater. 10 (1), 2070004. doi:10.1002/ aenm.202070004

Takada, K. (2013). Interfacial nanoarchitectonics for solid-state lithium batteries. Langmuir 29 (24), 7538-7541. doi:10.1021/la3045253

Umeshbabu, E., Zheng, B., and Yang, Y. (2019). Recent progress in all-solid-state lithium-sulfur batteries using high Li-ion conductive solid electrolytes. Electrochem. Energy Rev. 2 (2), 199-230. doi:10.1007/s41918-019-00029-3

Wang, C., Liang, J., Jiang, M., Li, X., Mukherjee, S., Adair, K., et al. (2020). Interface-assisted in-situ growth of halide electrolytes eliminating interfacial challenges of all-inorganic solid-state batteries. Nano. Energy. 76, 105015. doi:10.1016/j.nanoen.2020.105015

Wang, L., Liu, D., Huang, T., Geng, Z., and Yu, A. (2020). Reducing interfacial resistance of a $\mathrm{Li}_{1.5} \mathrm{Al}_{0.5} \mathrm{Ge}_{1.5}\left(\mathrm{PO}_{4}\right)_{3}$ solid electrolyte/electrode interface by polymer interlayer protection. RSC Adv. 10 (17), 10038-10045. doi:10.1039/d0ra00829j

Wang, Y., Richards, W. D., Ong, S. P., Miara, L. J., Kim, J. C., Mo, Y., et al. (2015). Design principles for solid-state lithium superionic conductors. Nat. Mater. 14 (10), 1026-1031. doi:10.1038/nmat4369

Wen, L., Zhou, M., Wang, C., Mi, Y., and Lei, Y. (2016). Energy storage: nanoengineering energy conversion and storage devices via atomic layer deposition. Adv. Energy Mater. 6 (23), 1-26. doi:10.1002/aenm.201670132 
Wenzel, S., Leichtweiss, T., Krüger, D., Sann, J., and Janek, J. (2015). Interphase formation on lithium solid electrolytes-An in situ approach to study interfacial reactions by photoelectron spectroscopy. Solid State Ion. 278, 98-105. doi:10. 1016/j.ssi.2015.06.001

Woo, J. H., Trevey, J. E., Cavanagh, A. S., Choi, Y. S., Kim, S. C., George, S. M., et al. (2012). Nanoscale interface modification of $\mathrm{LiCoO}_{2}$ by $\mathrm{Al}_{2} \mathrm{O}_{3}$ atomic layer deposition for solid-state $\mathrm{Li}$ batteries. J. Electrochem. Soc. 159 (7), A1120-A1124. doi:10.1149/2.085207jes

Wood, K. N., Steirer, K. X., Hafner, S. E., Ban, C., Santhanagopalan, S., Lee, S.-H., et al. (2018). Operando X-ray photoelectron spectroscopy of solid electrolyte interphase formation and evolution in $\mathrm{Li}_{2} \mathrm{~S}-\mathrm{P}_{2} \mathrm{~S}_{5}$ solid-state electrolytes. Nat. Commun. 9 (1), 2490. doi:10.1038/s41467-018-04762-Z

Xia, S., Wu, X., Zhang, Z., Cui, Y., and Liu, W. (2019). Practical challenges and future perspectives of all-solid-state lithium-metal batteries. Chem 5 (4), 753-785. doi:10.1016/j.chempr.2018.11.013

Xiao, Y., Miara, L. J., Wang, Y., and Ceder, G. (2019a). Computational screening of cathode coatings for solid-state batteries. Joule 3 (5), 1252-1275. doi:10.1016/j. joule.2019.02.006

Xiao, Y., Wang, Y., Bo, S.-H., Kim, J. C., Miara, L. J., and Ceder, G. (2019b). Understanding interface stability in solid-state batteries. Nat. Rev. Mater. 5 (2), 105-126. doi:10.1038/s41578-019-0157-5

Xu, R., Han, F., Ji, X., Fan, X., Tu, J., and Wang, C. (2018). Interface engineering of sulfide electrolytes for all-solid-state lithium batteries. Nanomater. Energy 53, 958-966. doi:10.1016/j.nanoen.2018.09.061

Xu, J., Liu, L., Yao, N., Wu, F., Li, H., and Chen, L. (2019). Liquid-involved synthesis and processing of sulfide-based solid electrolytes, electrodes, and allsolid-state batteries. Mater. Today Nano. 8, 100048. doi:10.1016/j.mtnano.2019. 100048

Yersak, T., Salvador, J. R., Schmidt, R. D., and Cai, M. (2019). Hot pressed, fiberreinforced $\left(\mathrm{Li}_{2} \mathrm{~S}\right)_{70}\left(\mathrm{P}_{2} \mathrm{~S}_{5}\right)_{30}$ solid-state electrolyte separators for Li metal batteries. ACS Appl. Energy Mater. 2 (5), 3523-3531. doi:10.1021/acsaem. 9 b00290

Yu, C., Ganapathy, S., Eck, E. R. H., Wang, H., Basak, S., Li, Z., et al. (2017). Accessing the bottleneck in all-solid state batteries, lithium-ion transport over the solid-electrolyte-electrode interface. Nat. Commun. 8 (1), 1086. doi:10. 1038/s41467-017-01187-y

Yubuchi, S., Ito, Y., Matsuyama, T., Hayashi, A., and Tatsumisago, M. (2016). 5 V class $\mathrm{LiNi}_{0.5} \mathrm{Mn}_{1.5} \mathrm{O}_{4}$ positive electrode coated with $\mathrm{Li}_{3} \mathrm{PO}_{4}$ thin film for all- solid-state batteries using sulfide solid electrolyte. Solid State Ion. 285, 79-82. doi:10.1016/j.ssi.2015.08.001

Zhang, B., Weng, M., Lin, Z., Feng, Y., Yang, L., Wang, L. W., et al. (2020). Li-Ion cooperative migration and oxy-sulfide synergistic effect in $\mathrm{Li}_{14} \mathrm{P}_{2} \mathrm{Ge}_{2} \mathrm{~S}_{16-6 \mathrm{x}} \mathrm{O}_{\mathrm{x}}$ solid-state-electrolyte enables extraordinary conductivity and high stability. Small 16 (11), 1906374. doi:10.1002/smll.201906374

Zhang, J., Zhong, H., Zheng, C., Xia, Y., Liang, C., Huang, H., et al. (2018). Allsolid-state batteries with slurry coated $\mathrm{LiNi}_{0.8} \mathrm{Co}_{0.1} \mathrm{Mn}_{0.1} \mathrm{O}_{2}$ composite cathode and $\mathrm{Li}_{6} \mathrm{PS}_{5} \mathrm{Cl}$ electrolyte: effect of binder content. J. Power Sources 391, 73-79. doi:10.1016/j.jpowsour.2018.04.069

Zhang, W., Richter, F. H., Culver, S. P., Leichtweiss, T., Lozano, J. G., Dietrich, C., et al. (2018). Degradation mechanisms at the $\mathrm{Li}_{10} \mathrm{GeP}_{2} \mathrm{~S}_{12} / \mathrm{LiCoO}_{2}$ cathode interface in an all-solid-state lithium-ion battery. ACS Appl. Mater. Interfaces 10 (26), 22226-22236. doi:10.1021/acsami.8b05132

Zhang, Z., Zhang, J., Sun, Y., Jia, H., Peng, L., Zhang, Y., et al. (2020). $\mathrm{Li}_{4}-\mathrm{Sb} \mathrm{Sn}_{1}-\mathrm{S}_{4}$ solid solutions for air-stable solid electrolytes. J. Energy Chem. 41, 171-176. doi:10.1016/j.jechem.2019.05.015

Zhao, F., Liang, J., Yu, C., Sun, Q., Li, X., Adair, K., et al. (2020). A versatile Snsubstituted argyrodite sulfide electrolyte for all-solid-state Li metal batteries. Adv. Energy Mater. 10 (9), 1903422. doi:10.1002/aenm.201903422

Zhu, Y., He, X., and Mo, Y. (2016). First principles study on electrochemical and chemical stability of solid electrolyte-electrode interfaces in allsolid-state Li-ion batteries. J. Mater. Chem. 4 (9), 3253-3266. doi:10. $1039 / \mathrm{c} 5 \mathrm{ta} 08574 \mathrm{~h}$

Conflict of Interest: The authors declare that the research was conducted in the absence of any commercial or financial relationships that could be construed as a potential conflict of interest.

This work is authored by Teerth Brahmbhatt, Guang Yang, Ethan C. Self and Jagit Nanda on behalf of the U.S. Government and, as regards Dr. Brahmbhatt, Dr. Yang, Dr. Self, Dr. Nanda, and the U.S. Government, is not subject to copyright protection in the United States. Foreign and other copyrights may apply. This is an open-access article distributed under the terms of the Creative Commons Attribution License (CC $B Y)$. The use, distribution or reproduction in other forums is permitted, provided the original author(s) and the copyright owner(s) are credited and that the original publication in this journal is cited, in accordance with accepted academic practice. No use, distribution or reproduction is permitted which does not comply with these terms. 